\title{
El papel del estudiante de medicina ante la pandemia de COVID-19. Una responsabilidad compartida
}

\author{
The role of the medicine student in COVID-19 pandemic. A shared responsibility
}

\begin{abstract}
Jorge E. Valdez-García1, Irma E. Eraña-Rojas², José A. Díaz Elizondo³, Mary A. Cordero-Díaz', Alejandro Torres-Quintanilla², Ramón I. Esperón-Hernández y Lydia Zeron-Gutiérrez * *

${ }^{1}$ Escuela de Medicina y Ciencias de la Salud, Tecnológico de Monterrey, Consejo Directivo, Asociación Mexicana de Facultades y Escuelas de Medicina (AMFEM), Monterrey, Nuevo León; ' ${ }^{E}$ scuela de Medicina y Ciencias de la Salud, Tecnológico de Monterrey, Monterrey, Nuevo León; ${ }^{3}$ Departamento Regional de Ciencias Clínicas, Tecnológico de Monterrey, Monterrey, Nuevo León; ${ }^{4}$ Programa de Postgrado Escuela de Medicina y Ciencias de la Salud, Tecnológico de Monterrey, Monterrey, Nuevo León; ${ }^{5}$ Facultad de Medicina de la Universidad Westhill, Consejo Directivo Asociación Mexicana de Facultades y Escuelas de Medicina (AMFEM), Ciudad de México; ${ }^{6}$ Escuela de Medicina y Ciencias de la Salud, Tecnológico de Monterrey, Ciudad de México. México
\end{abstract}

La declaración de pandemia de COVID-19 de la Organización Mundial de la Salud el 11 de marzo de 2020 ha obligado a ver con una nueva perspectiva el proceso formativo de los estudiantes de ciencias de la salud, entre ellos de la medicina, lo que ha generado una alteración completa de la operación que conocíamos como normal de las facultades y escuelas de medicina y ciencias de la salud, en todo el mundo, y ha provocado una disrupción de la educación médica'.

Todo cambió y no tenemos acceso a nuestras aulas, laboratorios, centros de simulación y comunidades, y mucho menos al principal escenario formativo, el clínico. Los centros de salud de primer nivel y los hospitales de segundo y tercer nivel han quedado limitados al acceso de los estudiantes, pues su prioridad en este momento son los pacientes que presentan el nuevo virus y los que sufren todas las demás enfermedades. Hoy, la virtualidad es el único escenario seguro',2.

A diferencia de otras disciplinas, tanto los profesores como los estudiantes tienen un papel activo en las labores propias de la profesión. Por lo tanto, es momento de replantear el papel que desempeñan los profesionales de la salud en formación, no solo para mantener la continuidad de sus procesos de aprendizaje, sino también para convertirse en un agente que forme parte de la respuesta sanitaria sin poner en riesgo su seguridad y la de los pacientes ${ }^{3}$.

Este compromiso de las instituciones educativas en salud de formar para ayudar ante la situación comprende, por un lado, la necesidad de continuar la formación de profesionales de salud con otras modalidades educativas que protejan la integridad y la seguridad de los involucrados, y por otro, el deseo de sumar esfuerzos para atender el incremento de las necesidades sanitarias derivadas de la pandemia ${ }^{4}$.

La Asociación Mexicana de Facultades y Escuelas de Medicina $^{5}$ y la Association of American Medical Colleges $^{6}$ se pronunciaron recomendando temporalmente la suspensión de actividades clínicas en ámbitos hospitalarios para los estudiantes de medicina y de ciencias de la salud que cursan el nivel de licenciatura. La necesidad de impedir el acceso a los estudiantes al entorno clínico hospitalario se debe al cambio inminente en las condiciones de atención, seguridad del paciente y limitación de recursos para protección personal en los sistemas de salud.

\footnotetext{
Correspondencia:

*Lydia Zeron-Gutiérrez

Del Puente, 222

Col. Ejidos de Huipulco, Del. Tlalpan

Fecha de recepción: 30-04-2020

C.P. 14380 , Ciudad de México, México

E-mail: lydia.zeron@tec.mx

Fecha de aceptación: 04-05-2020

DOI: 10.24875/CIRU.M20000066

0009-7411/C 2020 Academia Mexicana de Cirugía. Publicado por Permanyer. Este es un artículo open access bajo la licencia CC BY-NC-ND (http://creativecommons.org/licenses/by-nc-nd/4.0/).

Cir Cir. 2020;88(4):399-401 Contents available at PubMed www.cirugiaycirujanos.com
} 
La operación de los hospitales, al saturar sus servicios debido a la atención de pacientes relacionada con la pandemia de COVID-19, se vuelve compleja, y los estudiantes en ciclos clínicos o de internado aún no cuentan con las competencias necesarias para integrarse en los equipos del personal de salud que participa en la atención de los pacientes. Esta limitación de los estudiantes es otro factor que impide incorporarlos durante las etapas tempranas de la crisis, que se suma al relacionado con la seguridad del paciente, de los equipos de salud y del propio estudiante. Además, también existe una limitación de los centros hospitalarios para dedicarse a las actividades académicas habituales, como brindar atención y tutoría a los estudiantes, dado que ante esta compleja situación la prioridad son los pacientes y tanto los recursos humanos como los materiales (equipos de protección personal, equipos e insumos médicos, entre otros) tienen que estar disponibles y destinados a este propósito.

Durante las crisis sanitarias, como la pandemia actual de COVID-19, es indispensable generar un plan de acción que considere la diversidad de necesidades educativas de los profesionales en formación, de acuerdo con su etapa formativa.

En atención a las recomendaciones de las autoridades sanitarias y a las disposiciones oficiales, la suspensión de las actividades presenciales garantiza la protección de los estudiantes en sus primeros años de formación. Por tanto, la transformación a un modelo educativo a distancia permite la continuidad académica, de forma parcial o completa según los recursos disponibles.

Por todo lo anterior, se sugieren las siguientes actividades para los estudiantes de ciencias básicas: apoyo en la diseminación de información científica que contribuya al bienestar nutricional, físico y emocional durante la contingencia, crear equipos de apoyo para sus compañeros que mitiguen el impacto físico y social que la situación de aislamiento produce, y grupos de discusión clínica a través de actividades en línea.

En su artículo «Me preparo para ayudar: respuesta de escuelas de medicina y ciencias de la salud ante COVID19", Valdez, et al. ${ }^{4}$ mencionan que, dependiendo de la etapa de formación de los estudiantes, es diferente el grado de responsabilidad y de toma de decisiones que estos guardan en un entorno clínico. Por ello, el análisis de la participación de los estudiantes durante la contingencia deberá realizarse basándose en el grado académico y el nivel de dominio de competencias clínicas que presenten. La participación de los estudiantes de ciencias clínicas podrá desarrollarse si se cuenta con: 1) disponibilidad de equipo de protección personal; 2) protección garantizada, vigilancia/monitoreo del bienestar integral; y 3) en caso de ser requerida, atención médica. Es importante que el nivel de participación de los estudiantes en una etapa avanzada de formación clínica sea supervisado y tutorado por un experto clínico. Su participación es en gran medida como observador del proceso de atención sin obstaculizar la dinámica de la atención. El estudiante podrá guiarse del role model siguiendo el actuar de su tutor y de los expertos que se encuentran atendiendo pacientes potencialmente infectados; deberá seguir un algoritmo de observación de patrones clínicos, para identificar el mecanismo de razonamiento clínico que le lleve a tomar decisiones en cuanto a diagnóstico y medidas terapéuticas. Es importante considerar que, de acuerdo con la gravedad de la crisis sanitaria, el personal en formación se convierte en una reserva profesional que podría entrar en acción ante la emergencia nacional si se presenta una escasez de personal de salud $^{4}$.

En dicho escenario, o en caso de que la regulación de las entidades gubernamentales 0 institucionales impida que los estudiantes sean separados de las áreas de atención clínica, la institución que sostenga dicho impedimento tendrá que acatar la responsabilidad de la preparación y la seguridad de los estudiantes para la atención de pacientes, y deberá garantizar a los estudiantes la capacitación, el equipamiento de protección personal y la aplicación de protocolos que les permitan participar de forma segura y eficiente, tanto para ellos como para otras personas con quienes interaccionen (pacientes, personal de salud, compañeros y familiares). Es momento además para que los profesores desarrollen e implementen innovaciones educativas durante su ejercicio docente, y sean un modelo a seguir por sus estudiantes.

Por lo tanto, para los estudiantes de pregrado (estudiantes de ciclos clínicos y médicos internos de pregrado) y para los estudiantes médicos pasantes en servicio social, la Asociación Mexicana de Facultades y Escuelas de Medicina (AMFEM) solicita firmemente que se cumplan las siguientes recomendaciones y medidas:

- Retiro de las áreas de tamizaje, urgencias, críticas y/o especializadas para la atención de potenciales infectados de COVID-19, o de cualquier otro escenario clínico de riesgo. 
- Equipo de protección personal adecuado sin hacer distinción con el personal de salud adscrito.

- Capacitación formal para la autoprotección, así como protocolos de procedimientos claros para la detección de casos de COVID-19.

- Garantía de atención de su salud, y en caso de necesario de las secuelas de una posible infección.

- Retiro de estudiantes con factores de riesgo.

- Supervisión de la salud mental a través de acompañamiento y atención psicológica.

- Líneas abiertas de comunicación con autoridades de enseñanza institucionales y universitarias, atendiendo a las necesidades particulares que se pudieran presentar.

La desatención a estas directrices haría inviable la presencia de estudiantes en estos escenarios clínicos debido al incremento en el riesgo, tanto para ellos y sus entornos familiares como para los pacientes.

Enfatizamos la necesidad de reconocer la responsabilidad compartida entre las instituciones de salud y educativas en la supervisión del cumplimiento de todas las recomendaciones que se plantean para la incorporación de estudiantes de medicina de pregrado a ambientes clínicos con la finalidad de colaborar durante la pandemia garantizando su seguridad y la de sus entornos.

\section{Agradecimientos}

Los autores agradecen al Comité Ejecutivo de Asociación Mexicana de Facultades y Escuelas de Medicina (AMFEM) por sus observaciones al presente documento, así como a la presidencia de la Asociación Mexicana de Médicos en Formación (AMMEF) y a la coordinación del Colectivo de Médicos en Formación por sus comentarios a versiones previas.

\section{Bibliografía}

1. Eva KW. Strange days. Med Educ. 2020 Mar 27. doi: 10.1111/ medu.14164. [Epub ahead of print]

2. Woolliscroft JO. Innovation in response to the COVI-19 pandemic crisis. Acad Med. 2020 Apr 8. doi: 10.1097/ACM.0000000000003402. [Epub ahead of print]

3. Bauchner H, Sharfstein J. A bold response to the COVID-19 pandemic: medical students, national service, and public health. JAMA. $2020 \mathrm{Apr} 8$. doi: 10.1001/jama.2020.6166. [Epub ahead of print]

4. Valdez V, López M, Jiménez M, Díaz Elizondo JA, Dávila Rivas JA, Olivares S. Me preparo para ayudar: respuesta de escuelas de medicina y ciencias de la salud ante COVID-19. Inv Ed Med. 2020;9:1-11. (Consultado el 22 de abril de 2020.) Disponible en: http://riem.facmed.unam. $\mathrm{mx} /$ sites/all/archivos/prensa/COVID-19_RIEM.pdf

5. Asociación Mexicana de Facultades y Escuelas de Medicina. Comunicado importante Covid-19. Amfem.edu.mx . 2020. (Consultado el 25 de marzo de 2020.) Disponible en: http://www.amfem.edu.mx/index.php/ acerca/comunicados

6. Whelan A, Prescott J, Young G, Catanese V. Guidance on medical students' clinical participation: effective immediately. Association of American Medical Colleges. 2020. (Consultado el 25 de marzo de 2020.) Disponible en: https://www.aamc.org/system/files/2020-03/Guidance\%20 on\%20Student\%20Clinical\%20Participation\%203.17.20\%20Final.pdf 